\title{
PRESENTACIÓN \\ La gobernanza mundial y los debates sobre la seguridad alimentaria
}

\author{
Humberto González Chávez
}

$\mathrm{D}$ esde su creación en 1945, la Organización de las Naciones Unidas para la Agricultura y la Alimentación (FAO) ha tenido como preocupación central abatir el hambre y prevenir las crisis alimentarias a escala mundial mediante el mejoramiento de la agricultura, la ganadería y la pesca. Su planteamiento original fue que este problema se debe enfrentar con acciones coordinadas mundialmente y no sólo por los gobiernos de los Estados nacionales que lo padecen.

La FAO ha tenido capacidad de convocatoria a escala internacional y ha ocupado un lugar preeminente en la definición de las políticas agrícolas y alimentarias globales. Tres han sido los factores que explican este resultado (Phillips e Ilcan, 2003: 434-435). En primer lugar, fue un acierto concebir el problema del hambre y la nutrición en relación directa con la producción de alimentos; de esta manera, cualquier consideración o acción concreta para aliviar el primero debía considerar el estado de la agricultura, la ganadería y la pesca. En segundo lugar, históricamente la FAO se constituyó y ha justificado su actividad como una institución que podía actuar en un ámbito global con mayor legitimidad y justificación que otras instituciones internacionales, como la Organización Mundial del Comercio (OMC) (antes Acuerdo General de Aranceles Aduaneros y Comercio, GATT), el Banco Mundial (BM), el Fondo Monetario Internacional (FMI) o las grandes corporaciones trasnacionales, que no cuentan con igual enfoque humanitario en los objetivos que persiguen ni tienen la misma transparencia y rendición de cuentas que la FAO. Finalmente, desde su creación, este organismo ha ganado una legitimidad que le ha permitido actuar globalmente y acordar con los gobiernos nacionales acciones específicas para el mejoramiento del sistema agrícola y alimentario mundial. Esta posibilidad se presentó en el contexto de la posguerra, cuando había escasez de alimentos en Europa, lo que llevó a la temprana convicción de que había que reorganizar el sistema alimentario internacional por encima de los intereses particulares de los gobiernos nacionales y otorgar a la FAO un papel sobresaliente para coordinar los esfuerzos encaminados a lograr esta meta. Esta convicción se mantuvo y fortaleció, no obstante haberse superado la crisis alimentaria de la posguerra en Europa.

Estos tres factores llevan a Phillips e Ilcan (2003: 437) a plantear la tesis de que la preponderancia y el fortalecimiento de la FAO se explican debido a la instauración de mecanismos de gobernanza mundial que implicaron la elaboración y difusión de formas válidas de conocimiento para concebir la problemática agroalimentaria a nivel internacional, nacional, local y doméstico, las cuales sirvieron de referentes a los análisis más particulares; al desarrollo de formas de comunicación e interacción social

World Governance and the Debate on Food Security

HuMBERTO GONZÁLEZ CHÁVEZ: Centro de Investigaciones y Estudios Superiores en Antropología Social-Occidente, Guadalajara, Jalisco, México.

hgc@ciesas.edu.mx

Desacatos, núm. 25, septiembre-octubre 2007, pp. 7-20. 


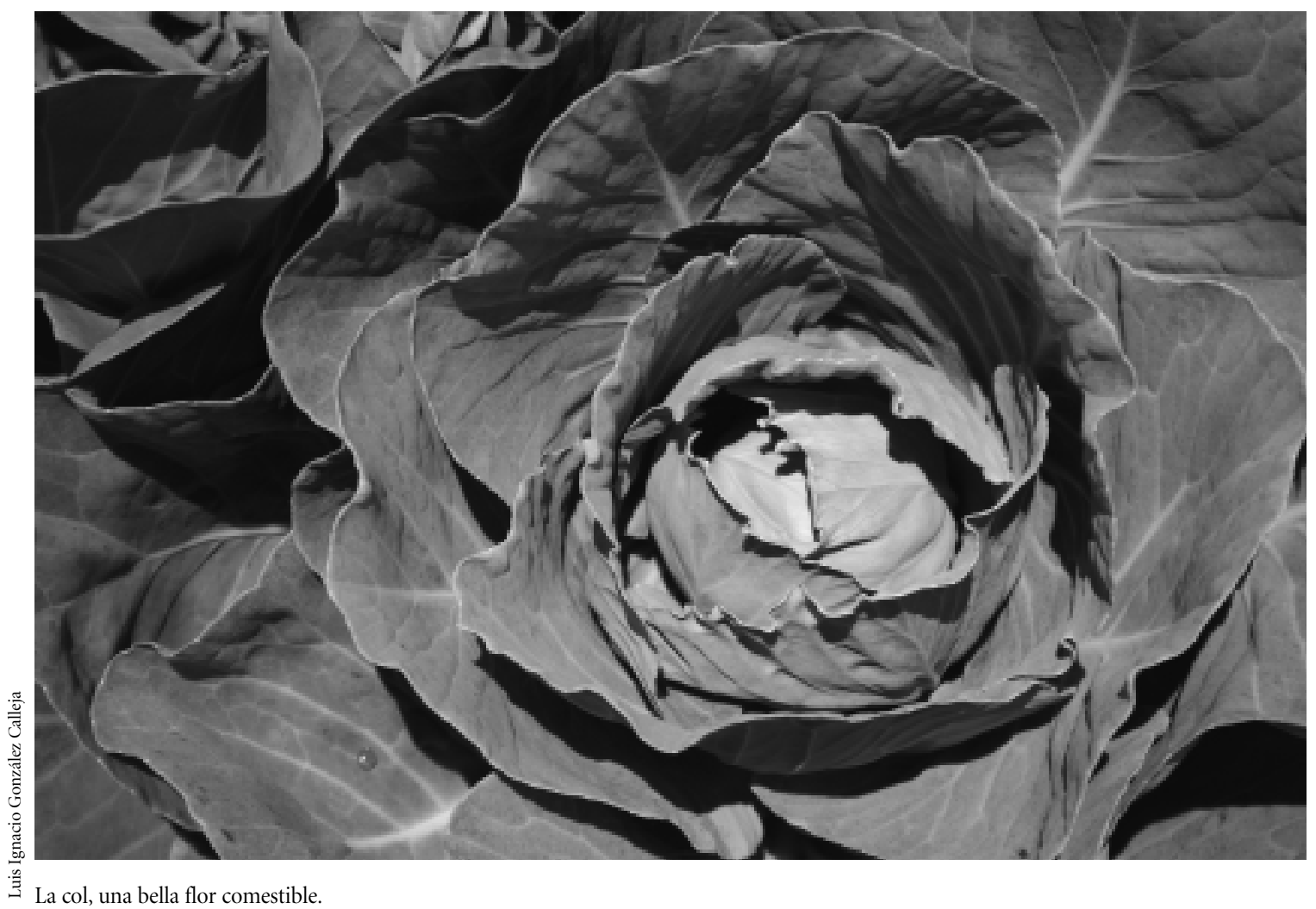

y redes de relaciones que permitieron establecer diversas maneras de coordinación, regulación y cooperación entre los gobiernos y entre las organizaciones públicas y privadas; finalmente, éstas llevaron a establecer programas y planes de acción que incidieron sobre la situación agroalimentaria global.

Este proceso de cambio alentado por la FAO se desarrolló a partir de una revalorización del conocimiento científico como herramienta fundamental para plantear iniciativas que permitieran mejorar la producción alimentaria y la nutrición; pero también, como recurso para justificar las propuestas de la FAO, particularmente las que llevaron a estandarizar ciertas prácticas y políticas a escala mundial (ibidem).

El concepto de seguridad alimentaria (SA) fue definido y adoptado por la Conferencia Mundial sobre la Alimentación realizada en Roma en 1974 y se convirtió en el propósito central de la FAO (FAO, 1975), pero también en el compromiso fundamental que contrajeron los gobiernos que firmaron el documento final de la conferencia y en el tema de debate de diversas organizaciones e instituciones públicas y privadas interesadas en la agricultura y la alimentación que incidían a escala global y local.

\section{AMBIGÜEDADY DISPUTAS POR EL ALCANCE Y SIGNIFICADO DE LA SEGURIDAD ALIMENTARIA}

El concepto de SA ha tenido más de doscientas definiciones (Smith, Pinting y Maxwell, 1992) y ha generado numerosos debates desde su adopción por la FAO. Las ambigüedades y controversias que se han suscitado tienen su origen en los múltiples criterios de análisis empleados para estudiar la compleja y heterogénea situación agroalimentaria mundial; en las diversas maneras en que la 
gente pobre del mundo experimenta la inseguridad alimentaria (Maxwell, 1996); y finalmente, como tendremos oportunidad de documentarlo más adelante, en la confrontación de intereses entre los distintos gobiernos y actores políticos y económicos, entre los que destacan las corporaciones trasnacionales, que buscan la no afectación de sus intereses por una institución que desempeña un papel relevante en la gobernanza agroalimentaria mundial.

Con base en documentos de la FAO publicados entre 1974 y 2003 sobre el contexto histórico y las preocupaciones científicas predominantes en ese periodo, Mechlem (2004) hace una biografía del concepto de SA. En primer lugar, encuentra que durante la década de 1970 el énfasis de la SA se dio en la necesidad de garantizar un abasto permanente de alimentos para la población mundial, en crecimiento, que permitiera hacer frente a las fluctuaciones anuales de la producción y la inestabilidad de los precios en el mercado mundial. Este énfasis, como lo señalé anteriormente, estaba en consonancia con el contexto de crisis en la producción agropecuaria y los precios altos que alcanzaron los alimentos en aquellos años. En este contexto prevaleció la concepción de que el problema del hambre - particularmente preocupante debido al crecimiento de la población mundial— se podía resolver con una mayor producción de alimentos (Falcon et al., 2005: 1117).

En segundo lugar, con las publicaciones de los trabajos de Amartya Sen en la década de 1980 se presentó un cambio radical en la tesis vigente sobre la SA en la FAO y fuera de ella. Este autor centra el problema de la inseguridad alimentaria en el acceso a los alimentos; las hambrunas — como él señala—ocurrían sin que hubiera una escasez significativa en los inventarios de alimentos. Por otra parte, podía haber disponibilidad y estabilidad en la oferta de alimentos y, a la vez, pobreza y hambre por falta de acceso a ellos. Esta situación explica que en 1983 la FAO enfatizara que debía haber "un acceso seguro a la oferta de alimento para aquellos que los necesitan" (Mechlem, 2004: 633). El mismo autor señala que, en este contexto, la discusión se centró en una definición más amplia de "subsistencia", la cual debía analizarse en el contexto del grupo doméstico en el que se organiza el consumo, se desarrollan actividades productivas y se maneja el ingreso, además de considerar las relaciones de cooperación y la jerarquía de poder entre los individuos que lo componen según la edad y el género. Esto último se debe a que diversas investigaciones sacaron a la luz que no había igualdad de acceso a los alimentos entre los individuos que componen el grupo doméstico, y que los más afectados eran las mujeres, los niños y los ancianos, debido al control que tenían los varones adultos sobre la producción y los ingresos familiares.

$\mathrm{Al}$ analizar la SA en el marco del grupo doméstico se presenta un cambio en su concepción, ya que de ser una problemática de carácter macro que atañe particularmente a los gobiernos nacionales y a la comunidad internacional, pasa a ser concebida en una dimensión microsocial, en la que se da cabida a la capacidad de decisión de los individuos que componen esta unidad social básica. Además, en ella se perfila la compleja interrelación e interdependencia entre los integrantes de la unidad doméstica, pero también de ésta con la comunidad de la que forma parte, con la nación y con el contexto internacional (Maxwell, 1996: 157).

En tercer lugar, la discusión sobre SA se reformuló debido a las investigaciones sobre salud y nutrición de las décadas de 1980 y 1990. Se planteó la relación entre una insuficiente e inadecuada nutrición y los problemas de salud de la población. Asimismo, se encontró que una mala nutrición tenía implicaciones en el desarrollo físico y mental de la población infantil. Por ello, al hablar de SA, la FAO prestó atención a la nutrición, además de otros factores relacionados con ella, como la higiene y la inocuidad de los alimentos (que estuvieran libres de patógenos).

Esta discusión se realiza en el marco de la unidad doméstica. Hay, por una parte, quienes ponen mayor énfasis en la organización y en las estrategias colectivas para subsistir y reproducirse y que conceden una atención central a la salud materna e infantil. Por el otro lado están quienes dan prioridad a las jerarquías de poder y a los conflictos y que plantean una atención más individualizada (Maxwell, 1996: 157). Aun y cuando se incorpora la discusión con base en el grupo doméstico, es claro que los textos de la FAO están muy lejos de una concepción que dé cuenta de la diversidad y complejidad que existe en la composición, organización y jerarquía interna de las uni- 
dades domésticas (Pottier, 1999: 14). En los documentos de la FAO predomina la idea de una organización familiar nuclear (compuesta de padre, madre e hijos), en la que no hay cabida para estructuras familiares diferentes, con múltiples acuerdos intradomésticos y con diferentes formas de vincularse al mercado y a la sociedad (ibid.).

Finalmente, la discusión sobre la SA incorporó el factor cultural al considerar la importancia que tienen en la alimentación las "preferencias alimentarias". Además de la suficiencia, la calidad nutritiva y el acceso a los alimentos, debía considerarse la diversidad de opciones alimenticias con que cuenta la población de acuerdo con sus tradiciones culinarias y los recursos ambientales y económicos a su disposición.

En 1996, como resultado de la amplitud y complejidad que adquirió el concepto de SA, la FAO propuso la siguiente definición en el documento World Food Summit: "La seguridad alimentaria, a nivel individual, familiar, nacional y regional, implica lograr que la población tenga acceso física y económicamente a una alimentación suficiente, sana y nutritiva, de acuerdo con sus preferencias, y que le permita satisfacer sus necesidades nutricionales y preferencias alimenticias para un vida activa y saludable" (cit. en Mechlem, 2004: 636, trad. propia).

A la pregunta sobre qué tan en serio se considera y acepta la diversidad cultural en la FAO, Pottier (1999: 15) responde, después de analizar diferentes documentos y programas de acción de esta institución, que se trata de un "gesto simbólico", ya que los puntos de vista de quienes viven la inseguridad alimentaria en realidad no se toman en cuenta, pues se perciben en una jerarquía inferior y sólo se seleccionan aquellos elementos que son de utilidad o que están relacionados con la política, pero descontextualizándolos del marco social y cultural en el que se encuentran. No obstante estas características, la definición de SA adoptada por la FAO en 1996 ha resultado un punto de referencia en el debate y en la elaboración y aplicación de políticas agroalimentarias por parte de los gobiernos nacionales. Cada uno, ante los otros gobiernos que firmaron el documento y ante diversos actores nacionales e internacionales, adquirió compromisos para definir su política agrícola y alimentaria. Ésta, al igual que las propuestas de la FAO misma, se elaboraban a partir de ciertas concepciones del desarrollo agroalimentario que permitían conocer la realidad económica, política y social en la que iban a ser aplicadas y prever determinados resultados a ser alcanzados.

\section{LAS IDEOLOGÍAS DEL DESARROLLOY LOS INTERESES ECONÓMICOS}

Para lograr la credibilidad y cooperación de los gobiernos, la FAO — de acuerdo con Phillips e Ilcan (2003: 453)— desarrolló bases de información estadística, sistematizó y difundió una extensa información científica, basada en diversas disciplinas que estudian la producción, el mercado y el consumo de alimentos en el mundo. En esta plataforma fundamentan sus evaluaciones y propuestas de política alimentaria global. La puesta en operación de estas últimas, según los mismos autores, se ha apoyado en una metodología inductiva (scientific management) basada en principios de optimización y consistencia, mediante procedimientos racionales con una estructura lógica distinguible. De esta manera, se ha buscado alcanzar el consenso de los países miembros.

Esta forma de operar, sin embargo, ha llevado a la FAO a presentar sus textos en un lenguaje apolítico, en el que se plantean de forma retórica propuestas que no llegan a ser más que listas de buenas intenciones; además, en ellos se evita hacer referencia a las causas históricas, económicas y políticas que originan la pobreza y se trasluce una incapacidad y desinterés por atender la diversa y compleja realidad sociocultural local (Pottier, 1999: 17). En estos documentos los problemas del hambre y su solución quedan en el ámbito de las obligaciones y de la decisión de cada país, con lo que tácitamente se exime de responsabilidad a la "comunidad internacional" y se evita fijar posturas críticas y acciones específicas frente a las instituciones financieras que desarrollan mecanismos de gobernanza global — como el BM y el FMI-que repercuten en el desarrollo de la agricultura y la alimentación en el planeta. Asimismo, se evade la crítica a la creciente influencia de las corporaciones agroalimentarias multinacionales sobre los gobiernos y sobre la agricultura y la alimentación en el planeta. 
El lenguaje apolítico y retórico de la FAO no consigue ocultar las ideologías del desarrollo que han prevalecido en esta institución y que han permitido a los "elaboradores de políticas" (una élite burocrática) evaluar y actuar sobre la problemática agroalimentaria global. Este sector de la burocracia, como lo señalamos anteriormente, se ha valido de marcos conceptuales y de resultados de investigaciones aplicadas para analizar los procesos de cambio social global, nacional, local y doméstico y formular políticas de acción específicas. A estos referentes que sirven de base a las políticas, Grindeel (1986) los llama ideologías del desarrollo. Con base en ellas se definen y justifican los objetivos, estrategias, instrumentos y mecanismos de evaluación de las políticas, y se elaboran los argumentos para justificar los supuestos beneficios a alcanzar con las políticas — particularmente cuando su aplicación afecta los intereses de sectores o de grupos específicos de la población-y sus resultados, cuando éstos son insatisfactorios (ibid.; González, 2004 y 2005).

A la propuesta de esta autora me interesa agregar que la burocracia que elabora y evalúa las políticas realiza un proceso de apropiación y síntesis de los marcos analíticos de los que se vale para analizar la problemática del desarrollo en razón de la posición política en la que participa activamente; por ello, sus propuestas no pueden dejar de considerar los intereses prevalecientes, particularmente de los gobiernos, instituciones y grupos con mayor poder económico y político. Por lo mismo, el término de ideología del desarrollo incorpora elementos y se aplica en este trabajo a la concepción discursiva y práctica del sector burocrático responsable de las políticas.

Cuando una política es puesta en duda o rechazada por algún grupo o institución ajeno a la burocracia responsable de formularla o aplicarla, los argumentos sobre sus inconsistencias, equívocos o efectos perversos son el principal recurso para proponer su reformulación o sustitución y así lograr la cooperación o evitar la confrontación. El análisis crítico de la política se plantea con base en la misma ideología de la élite burocrática, o bien, se recurre a perspectivas de análisis que aportan nuevos elementos a la discusión o ponen en tela de juicio la consistencia y validez de las propuestas de esta élite. En el campo de las políticas alimentarias a escala mundial ha

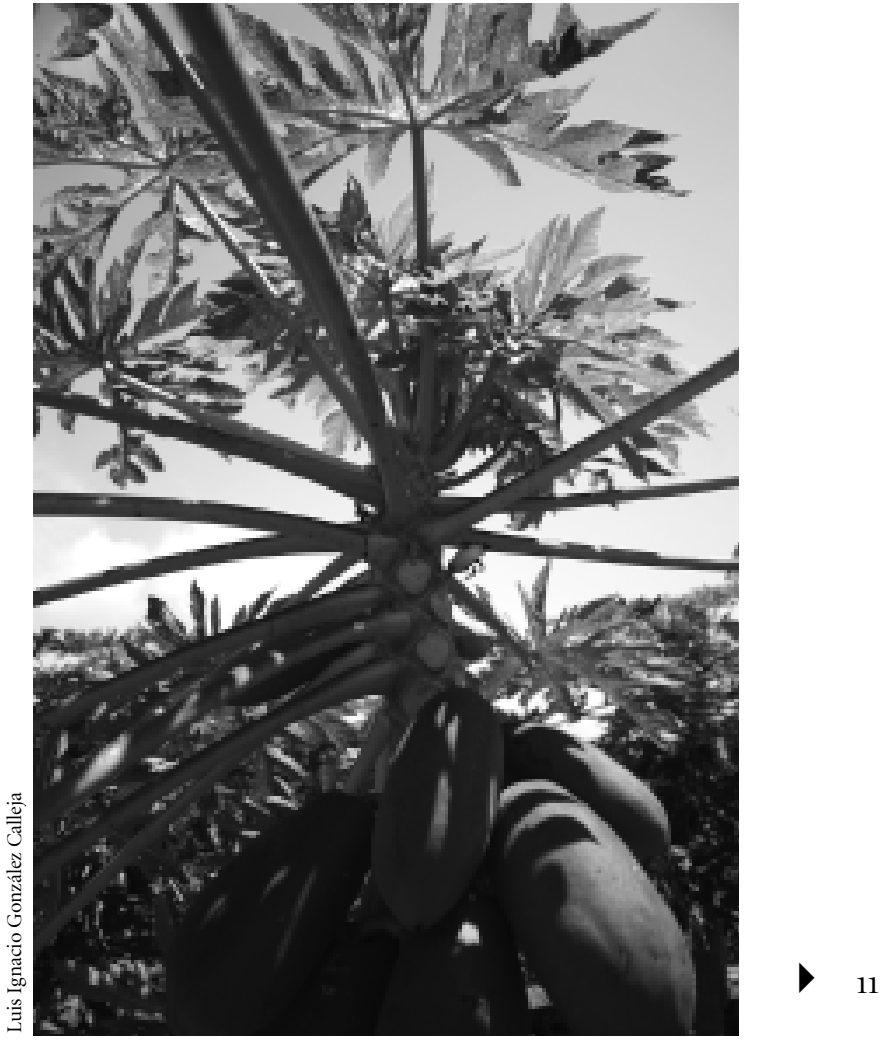

Papayo, fruta local y fruta exótica en el mercado transnacional.

habido un debate en torno a las ideologías prevalecientes de la FAO. Esta controversia se sustenta en criterios científicos; sin embargo, su discusión y negociación es política y adquiere la forma de una arena de lucha, en la que entran en juego diversos intereses económicos y geopolíticos de los países o de bloques de ellos.

Con base en una revisión y seguimiento de las actas de discusión previas a la firma de documentos de la FAO, planteo que la competencia y rivalidad ideológica en esta institución no desemboca en un "consenso ideológico"; antes bien, desencadena una lucha por alcanzar la hegemonía. De acuerdo con la propuesta de Roseberry (1994: 358), esta hegemonía no da por terminado el cuestionamiento y el rechazo de las contrapartes, ni cierra la posibilidad a nuevos desafíos teóricos e ideológicos por parte de quienes se ven afectados o excluidos con la formulación y la aplicación de las políticas. Las iniciativas 


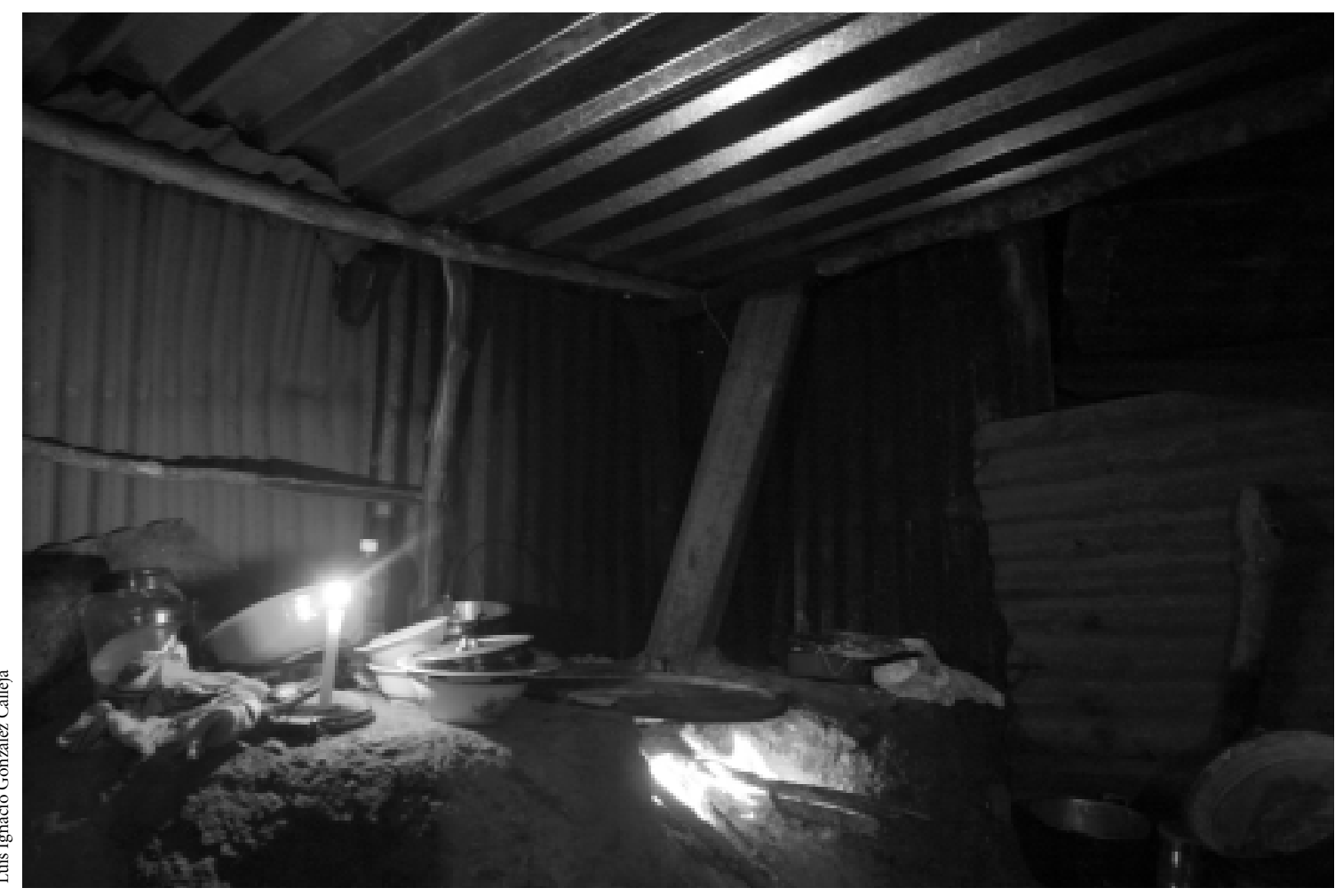

Producción de tortillas a la luz del fogón y de la vela.

para ganar fuerza y lograr una supremacía pueden ser múltiples (alianzas, coaliciones, confederaciones, etc.), así como los argumentos (éticos, políticos, económicos, prácticos y otros) y las justificaciones que entren en juego en esta arena de lucha ideológica.

En la década de 1980, en la FAO y en otras instituciones internacionales se presenta un cambio radical en la definición del papel que debía desempeñar la agricultura en el desarrollo de un país y en el de los gobiernos nacionales en relación con la seguridad alimentaria. Anteriormente, la agricultura era considerada un "sector estratégico" para la supervivencia de la población nacional y la estabilidad de la economía; esto último debido a que el alza de los precios en los alimentos tiene fuertes repercusiones en los salarios de los trabajadores y, por lo tanto, en los precios de las mercancías y la competitividad de la economía nacional (Fristcher, 2004: 115). Por ello, la concepción que prevalecía en aquel momento con- sideraba que un país debía ser autosuficiente y garantizar una oferta interna de alimentos al margen de las fluctuaciones de los precios en el mercado internacional y depender marginalmente de las importaciones (ibid.). Esta posición se sustentaba en la aceptación de que la producción agropecuaria está condicionada por factores naturales, los cuales son impredecibles y están fuera del control humano. Debido a esto, la agricultura, a diferencia de otros sectores de la economía, tenía una cláusula de "excepcionalidad" que permitía a los gobiernos nacionales proteger "legítimamente" a sus productores mediante la aplicación de medidas arancelarias y no arancelarias. También se aceptaba que el gobierno de un país mantuviera reservas de alimentos, estableciera precios de garantía, controlara la oferta interna de ellos y otorgara diversos tipos de subsidios a sus productores. De acuerdo con esta ideología, la SA se consideraba un asunto de seguridad nacional que debía ser garantizada primordial- 
mente por los Estados (Torres, 2003). De ahí el término de "soberanía alimentaria" que guió las políticas nacionales en diversos países —entre ellos México— hacia la "autosuficiencia alimentaria". Un análisis histórico sobresaliente de los elementos centrales — de carácter económico, ecológico, político y cultural- que entraron en juego en el desarrollo agrícola durante la época en la que se diseñó esta política podemos encontrarlo en el trabajo de Cynthia Hewitt en este número de la revista.

En la segunda mitad de la década de 1980 cobró vigencia una nueva ideología del desarrollo sobre la agricultura y la alimentación que Fristcher (2004) analiza en la Organización Mundial del Comercio, pero que también se presenta en la FAO y en otras instituciones internacionales. Desde el punto de vista de esta ideología emergente, el mercado mundial sería el principal garante de la seguridad alimentaria. Desde esta nueva óptica, las barreras arancelarias pasaron a ser vistas como un impedimento mayúsculo para incentivar la productividad agropecuaria y abaratar los precios de los alimentos en el mercado mundial. El concepto de autosuficiencia alimentaria fue sustituido por el de "autocapacidad" (ibid.: 118). Un país debía disponer de las divisas suficientes para comprar en el mercado mundial los alimentos necesarios para garantizar la alimentación de su población. La FAO misma definió un estándar para evaluar el riesgo en que una economía nacional incurre al carecer de las divisas suficientes para comprar los alimentos (Mendoza, 2000). De acuerdo con esta propuesta, un país entra en riesgo cuando el valor de sus importaciones alimentarias es mayor a $25 \%$ del valor de sus exportaciones totales. Este indicador es, por otra parte, equívoco para valorar el riesgo alimentario en el que cae una nación al depender de manera creciente de la importación de alimentos, ya que puede haber un aumento de la dependencia alimentaria y pasar desapercibido, debido a que hay un incremento de las exportaciones totales (Torres y Aguilar, 2003: 99). Bajo este nuevo parámetro ideológico, la SA pasa a considerarse en términos de las variables macroeconómicas de un país y se menosprecie el riesgo de escasez y encarecimiento de los alimentos al reducirlo a un sencillo monitoreo del mercado internacional y de la disponibilidad de divisas internas. En este número de $D e-$ sacatos, Archanjo et al. presentan un análisis de la producción y la disponibilidad de alimentos en América Latina y el Caribe en el contexto mundial y concluyen que hay una mayor dependencia y vulnerabilidad de esta región al requerir del mercado exterior para la obtención de los alimentos demandados por su población.

El debate en torno a la definición de las políticas agroalimentarias nacionales en el marco de una economía cada vez más globalizada se presentó abiertamente en la Organización Mundial del Comercio, en la que los presupuestos de la nueva ideología del desarrollo fueron muy discutidos e impugnados. En este contexto, en 1986 se organizó la primera Ronda de Uruguay, en la que los países miembros debatieron sobre la necesidad de limitar y regular los aranceles de los productos agropecuarios. A esta ronda siguieron otras con resultados limitados en cuanto a una liberalización comercial agropecuaria a escala global; los principales opositores de esta medida fueron las economías más desarrolladas: la Unión Europea, Estados Unidos y Japón (Torres y Aguilar, 2003; y Losch, 2004).

En la FAO, la hegemonía de la nueva ideología del desarrollo se hizo evidente en la Declaración de Roma en noviembre de 1996. En este documento se abandonó el enfoque sobre los alimentos básicos y se planteó en términos generales la discusión sobre el origen del problema alimentario mundial. No deja de ser significativo el silencio injustificado sobre el hambre y la desnutrición de la población más vulnerable de los países del tercer mundo que provocaron las políticas de ajuste estructural y la liberalización comercial (Pottier, 1999: 17). Una evaluación crítica de estas políticas en la vulnerabilidad alimentaria de los países en desarrollo se encuentra en los artículos de González y Macías y de Archanjo et al. (en este número), quienes analizan la situación particular de México y de Brasil, respectivamente, dentro de un contexto global. En el primer trabajo, el concepto de vulnerabilidad alimentaria se aplica no sólo al riesgo alimentario en un país que depende de manera creciente del mercado mundial para satisfacer las demandas alimentarias básicas, sino además a la degradación ambiental de los recursos naturales utilizados para producir alimentos, a la disminución de su riqueza genética — particularmente la desaparición de variedades originales de semillas- $-\mathrm{y}$ 
a la pérdida de saberes y tradiciones agroalimentarios que se presenta con la migración masiva de pequeños productores a las ciudades y a otros países.

Después de la cumbre de Roma, la Organización de las Naciones Unidas promovió reuniones y programas internacionales que buscaban comprometer a los gobiernos nacionales y a las organizaciones privadas y no gubernamentales en la lucha por disminuir el apremiante problema del hambre en el mundo. Con motivo del inicio del nuevo milenio, en la ciudad de Nueva York se organizó la Cumbre del Milenio, en la que participaron 191 gobiernos. El primero de los ocho objetivos que sería alcanzado en el año 2015 era erradicar la pobreza, lo que implicaba que dentro de 15 años debía reducirse en 50\% el número de personas con ingresos menores de un dólar al día. El optimismo y la euforia del milenio daban por sentado que un número reducido de objetivos y metas cuantificables en un periodo de tiempo que se antojaba razonable daría como resultado un compromiso y un esfuerzo colectivo mayor de los gobiernos y los organismos internacionales. Este supuesto, sin embargo, se puso en tela de juicio en la segunda Cumbre Mundial sobre la Alimentación, efectuada en 2002 en la misma ciudad de Roma, en la que se analizó el problema del hambre, la pobreza y el compromiso de la "comunidad internacional" pasados cinco años de la Cumbre de 1996. En sus intervenciones, el director general de la FAO, Jacques Diouf, planteó sin ambages:

Las personas hambrientas son casi tan numerosas hoy como lo eran hace cinco años. Esa es la triste realidad que nos indujo a convocar la Cumbre Mundial sobre la Alimentación cinco años después.

No se han cumplido las promesas y, lo que es peor, los hechos contradicen las palabras. Desgraciadamente la voluntad política y los recursos financieros no han estado a la altura de la solidaridad humana.

La cifra de personas subnutridas ha disminuido solamente en 6 millones al año, y no en 22 millones, como sería necesario para lograr el objetivo de la Cumbre [fijado también para 2015]. A este paso, se conseguirá dicho objetivo con 45 años de retraso (FAO, 2002).

Las causas de estos famélicos resultados quedaron archivadas en las síntesis de las distintas mesas de discu- sión de los delegados, que bien pudieron haber justificado compromisos más eficaces de política agrícola y alimentaria global. Se identificaron como causas la insuficiente inversión de los gobiernos en el desarrollo de su agricultura; la desigualdad en los subsidios directos e indirectos de los gobiernos de los países desarrollados a sus productores y sus exportaciones; las barreras arancelarias y no arancelarias (obstáculos sanitarios y técnicos) que aplican los países desarrollados a los productos agrícolas y a los alimentos industrializados, que limitan las oportunidades para desarrollar la agricultura y la agroindustria de los países en vías de desarrollo; el deficiente apoyo tecnológico y financiero a los pequeños agricultores, quienes son obligados a competir en condiciones de gran desigualdad en sus mercados agrícolas nacionales abiertos al mercado internacional; los peligros que entraña una agricultura que no considera la utilización sustentable de los recursos naturales, la contaminación, la inocuidad de los alimentos, etc. A estas causas se sumaron los problemas que la FAO ha señalado desde sus orígenes, como la vulnerabilidad que enfrentan las regiones más pobres y los territorios insulares ante la escasez de alimentos.

No obstante los escasos resultados internacionales en la erradicación del hambre, el debate sobre la seguridad alimentaria continúa siendo una asignatura pendiente en la agenda internacional. En los ámbitos académico y político internacionales se discuten nuevos paradigmas que recogen las demandas de organizaciones nacionales e internacionales y de diversos grupos que buscan alternativas más eficaces para enfrentar la inseguridad alimentaria.

\section{NUEVOS PARADIGMAS SOBRE LA AGRICULTURAY LA ALIMENTACIÓN}

Hoy día, en la discusión sobre la SA podemos advertir diferencias entre los países desarrollados del norte y los del sur. En los primeros es urgente resolver el problema de la inocuidad de los alimentos que ha causado gran alarma en la población, particularmente desde que apareció la enfermedad de las vacas locas; asimismo, es necesario enfrentar los problemas de degradación ambiental de una agricultura que ha buscado incrementar la producción y 


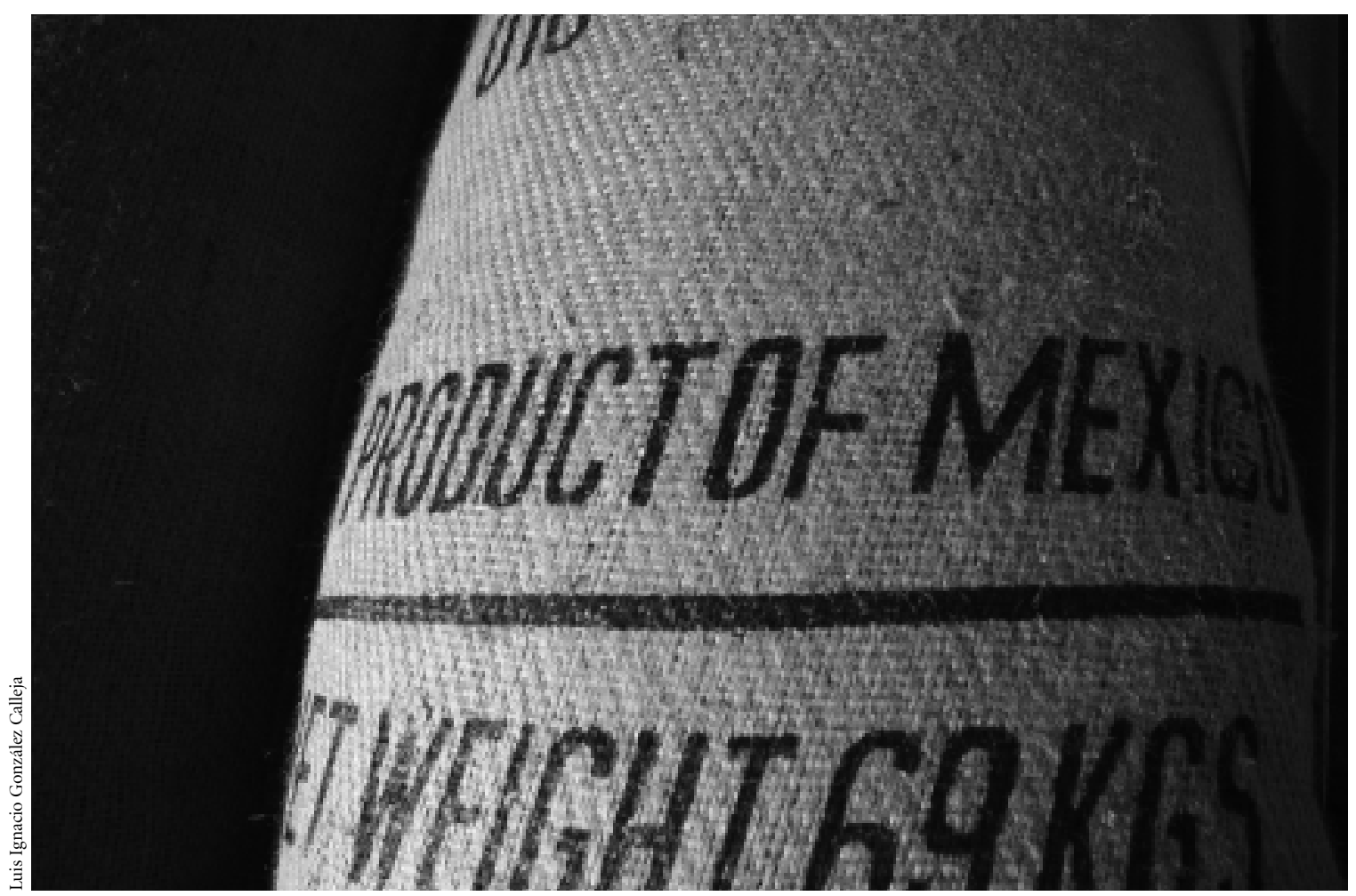

Café de exportación en Belisario Domínguez, Chiapas.

la competitividad como principal objetivo, sin considerar la producción de alimentos para las generaciones futuras; finalmente, destaca la búsqueda de alternativas económicas que permitan aligerar la pesada carga que representan los subsidios que los Estados nacionales otorgan a sus productores de alimentos. En el caso de la Unión Europea, además, se añade la preocupación por los cambios del paisaje agrícola y la pérdida del patrimonio cultural y alimentario que durante siglos desarrolló la población rural (Losch, 2004). En los países del sur se pone el énfasis en la creciente pobreza en las áreas rurales y urbanas, la cual se ha incrementado a raíz de las políticas de apertura comercial y desregulación económica que alentaron organismos internacionales como el Banco Mundial, el Fondo Monetario Internacional y los sectores internos más dinámicos y competitivos de la agricultura nacional asociados al gran capital transnacional (Sepúlveda, 2002). También surgen organizaciones y movimientos que in- tegran a campesinos, universitarios y organizaciones no gubernamentales preocupados por la disminución de la biodiversidad, la ampliación del uso y consumo de cultivos transgénicos y la degradación de los ecosistemas, particularmente, en las selvas tropicales.

Respecto a la arena política, en la que están en juego distintas ideologías y diversos intereses sobre la agricultura y la alimentación, me interesa comentar dos propuestas analíticas que cobran relevancia en la discusión actual y que han permeado las agendas políticas de organismos internacionales y de distintos países. La primera se ha desarrollado principalmente en Europa —en particular en Francia- y se ha identificado con el término de "multifuncionalidad de la agricultura” (MFA). Sus análisis y propuestas han sido considerados por la Unión Europea en la definición de la Política Agrícola Común, por países como Suiza, Noruega, Japón y Corea del Sur, y en foros internacionales en los que se discute la política agroali- 


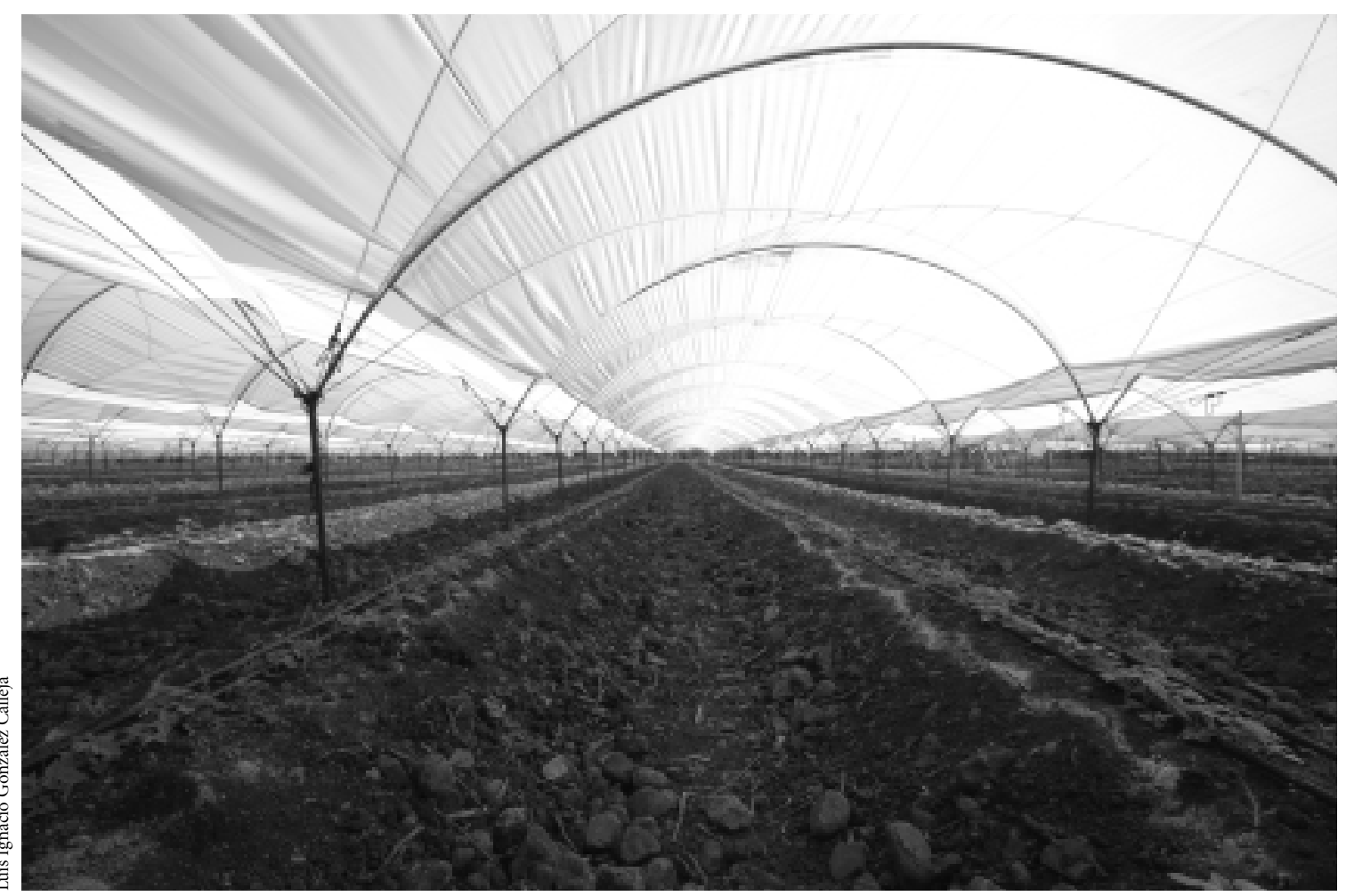

Agricultura bajo techo, Jocotepec, Jalisco.

mentaria, como la Conferencia sobre Medio Ambiente y Desarrollo en Río de Janeiro, en 1991 (Bonnal et al., 2003). La segunda se ha desarrollado en América Latina y se identifica con el nombre de "Nueva Ruralidad" (NR), con presencia en organismos latinoamericanos que influyen en la política agroalimentaria y en los países de América Latina (Instituto Interamericano de Cooperación para la Agricultura).

La MFA (Bonnal et al., 2000; Losch, 2002 y 2004; Drand y Van Huylenbroeck, 2003; Di lacobo, 2003) considera que la agricultura no sólo debe atender la tarea de producir alimentos e insumos industriales a precios bajos para satisfacer la demanda del mercado nacional y aprovechar las oportunidades que ofrece el mercado internacional, sino también considerar otras "funciones" igualmente trascendentales que están relacionadas con el territorio, los recursos naturales y las tradiciones y manifestaciones socioculturales; muchas de estas funciones no están valoradas en el mercado o se consideran bienes libres. Dentro de estas funciones cabe mencionar la preservación del paisaje agrícola, la multiplicación del empleo en las áreas rurales, la producción de alimentos saludables e inocuos para la población, la preservación de las tradiciones regionales agrícolas y culinarias que se pierden día a día con la disminución de la población rural; y finalmente, considera de manera prioritaria la sustentabilidad ambiental, que sólo se valora cuando se ve afectada de manera grave. La MFA sitúa las actividades agrícolas en el conjunto de la sociedad, sin reducir la agricultura a un sector económico al que hay que menospreciar porque genera una participación cada vez menor en el producto interno bruto nacional; asimismo, propone considerar la totalidad de las actividades y los servicios vinculados a las actividades productivas para tener una mejor comprensión de su importancia económica, social y cultural; plantea el fortalecimiento de la participa- 
ción de los actores y los gobiernos locales; y critica la posición de la economía neoclásica que considera como "externalidades negativas" del desarrollo agrícola la contaminación, el abandono rural, etcétera. La MFA también ha recibido críticas de quienes están a favor de una liberalización del comercio internacional y que consideran que esta ideología ha sido un recurso discursivo para justificar altos subsidios y mantener barreras proteccionistas que limitan el comercio mundial.

En América Latina, la NR ha abierto un debate frontal con la ideología y las políticas "neoliberales" adoptadas por la mayoría de los gobiernos latinoamericanos que ha llevado a revisarlas e implementar nuevas políticas (Echeverri, 2002 y 2006; Sepúlveda, 2002); sin embargo, esta posición crítica también ha sido retomada por autores que insisten en un cambio de rumbo y proponen alternativas para la ideología dominante en América Latina (Gordillo, 2003; Barkin, 2001). Sin pretender ser exhaustivo, la NR señala la necesidad de enfrentar las marcadas desigualdades regionales y pugna por un desarrollo territorial más equilibrado, que permita aprovechar las oportunidades que ofrecen los mercados regional, nacional e internacional; critica la dicotomía rural/urbano y la concepción según la cual las dinámicas de crecimiento deben de ser sectoriales; destaca la importancia de la sustentabilidad de la agricultura por encima de los criterios de competitividad que preocupan particularmente a la burocracia gubernamental; plantea que la lucha por abatir la pobreza rural debe buscar el fortalecimiento de la participación de los actores productivos y reforzar las medidas institucionales que permitan la cooperación de los actores públicos y privados en el campo; revaloriza las contribuciones de la producción tradicional y plantea un desarrollo participativo; propone afianzar la democracia y la gobernabilidad en las áreas rurales; por último, señala que es importante preservar y acrecentar la riqueza genética y la biodiversidad de los países latinoamericanos frente al modelo industrial dominante que ha generado una creciente concentración de la riqueza y la pobreza rural.

Las dos perspectivas tienen elementos comunes (Bonnal et al., 2003) en su crítica a un desarrollo agrícola que se ha concretado en buscar una mayor productivi- dad y competitividad agropecuaria. Ambas proponen revertir los cambios sobre la base de lograr dinámicas territoriales que permitan contrarrestar las desigualdades sociales y territoriales y alcanzar un desarrollo rural compartido y sustentable en el que se fortalezca la participación de los pequeños y medianos productores y la cooperación de instituciones públicas y privadas. Mientras que la MFA pone mayor énfasis en el papel regulador del Estado y de las políticas agrícolas comunes de los países miembros de la Unión Europea, la NR enfatiza las oportunidades que se presentan con el desarrollo regional sustentable, en el cual pueden lograrse sinergias locales entre las diferentes unidades productivas que permitirían conseguir economías de escala y reducir los costos de transacción (ibid.).

La MFA y la NR han enriquecido el debate de la SA en múltiples foros locales, nacionales e internacionales, han dado sustento y justificación a las propuestas de cambio en estos mismos ámbitos y permiten la difusión de experiencias positivas en materia de agricultura y alimentación. Frente a la ideología hegemónica que impera en las instituciones internacionales, se ha abierto un frente amplio global que participa en el debate sobre la SA desde una perspectiva ética y que ha establecido formas de comunicación y redes de relaciones que le permiten converger local y globalmente.

\section{UNA PROPUESTA UNIVERSAL A FAVOR DEL DERECHO A LA ALIMENTACIÓN}

En la discusión sobre la SA cobra fuerza el planteamiento de que la alimentación es un derecho humano que tiene su fundamento en la Declaración Universal de los Derechos Humanos de la Organización de las Naciones Unidas (ONU) de 1948. Explícitamente este derecho se incluyó en el Pacto Internacional de Derechos Económicos, Sociales y Culturales propuesto por la ONU en 1966 y que fue ratificado y firmado por los gobiernos miembros en 1976. En este pacto se consideró que el derecho a un nivel de "vida adecuado" abarca la alimentación, el vestido y la vivienda; sin embargo, en este documento no se profundizó en las implicaciones que tenía hablar de la ali- 
mentación adecuada como un derecho. Fue hasta 1985 que la ONU dio un paso más al crear la Comisión de Derechos Económicos, Sociales y Culturales (CDESC) para supervisar la aplicación de los derechos humanos entre los gobiernos de los países miembros. Esta comisión retomó la discusión que se había dado en torno a la SA en el seno de la FAO (Mechlem, 2004: 639) y propone que existe un "derecho de todos a tener en todo momento acceso físico y económico a una alimentación adecuada en cantidad y calidad y a los medios para procurársela" ${ }^{\text {. }}$

Plantear el derecho a la alimentación (DA) es reconocerle un valor intrínseco y darle mayor amplitud y obligatoriedad de la que hasta ese momento se le había dado (Mechlem, 2004: 644-645). El derecho a la alimentación se sustenta en el respeto a la dignidad humana y tiene, como cualquier otro derecho humano, un carácter universal, indivisible e interdependiente con el resto de los derechos ${ }^{2}$. Por ello, independientemente de las ideologías y sus sistemas políticos y económicos y de sus particularidades culturales, los Estados deben respetar, proteger y proveer los medios para garantizar el DA de su población.

Situar el problema de la alimentación en el marco de los derechos humanos tiene implicaciones muy concretas. Su cumplimiento implica obligatoriedad, transparencia y rendición de cuentas por parte de todos los gobiernos nacionales a la sociedad civil de sus naciones y del mundo; implica reconocer la vulnerabilidad de la población pobre y marginada, pero también sus potencialidades y recursos para procurarse una alimentación suficiente, nutritiva y sana (Mechlem, 2004: 646). Finalmente, el DA, como veremos más adelante, se ha convertido en un objetivo reconocido y válido universalmente, con implicaciones en la gobernanza alimentaria mundial.

El reconocimiento de que la alimentación es un derecho es, sin duda, el sustento a partir del cual los gobiernos, en la Cumbre Mundial sobre la Alimentación en Roma (FAO, 1996) y en la Declaración del Milenio33,

${ }^{1}$ CDESC, The Right to Adequate Food, E/C.12/1999/ 5, 12 de mayo de 1999.

${ }^{2}$ Conferencia Mundial de Derechos Humanos, Viena, 25 de junio de 1993. A/CONF.157, parte I, cap. III se comprometieron a reducir el hambre y la pobreza. Así como la discusión sobre la SA influyó en la definición del DA, así también esta discusión tuvo efectos sobre el cambio de postura unánime de los gobiernos en lo referente a su responsabilidad en la desnutrición crónica del mundo. Su falta de compromiso con las metas propuestas ha sido objeto de fuertes críticas, las cuales dan por sentado que se trata de un derecho universal.

Esta discusión ha tenido repercusiones en las políticas sociales de múltiples gobiernos nacionales, como es el caso de Brasil con su plan de "Hambre Cero" y en México con los planes de "Solidaridad", "Oportunidades" y "Progresa".

En los hechos, también, la universalidad del DA ha pasado a formar parte del interés y de los objetivos de trabajo de muy diferentes grupos y sectores sociales, políticos, religiosos y humanitarios a escala local y mundial. Ellos no solamente critican a sus gobiernos y a las instituciones internacionales sobre los magros alcances en esta materia, también han sido capaces de sobreponerse a sus diferencias y han desarrollado vínculos y creado convergencias para promover local, nacional y globalmente la cooperación y el desarrollo sustentable en grupos marginados, en los que el mejoramiento del ingreso y de la alimentación son parte importante de sus actividades. Dentro de estas organizaciones podemos señalar el movimiento a favor de un "comercio justo", en el que se articularon grupos religiosos y laicos y organizaciones ambientalistas (Aranda y Morales, 2002; VanderHoff, 2002; González, 2002; Nigh, 1997). Esta red de organizaciones presente en 21 países de diferentes continentes ofrece a las organizaciones solidarias de pequeños productores la oportunidad de comercializar sus productos en el mercado internacional a precios más altos que los convencionales; además, promueven el desarrollo de una producción orgánica y sustentable. Frente a aquellas ideologías que sólo reconocen como motor del desarrollo el interés individual y la rentabilidad económica, este tipo de iniciativas se sustenta en otras ideologías y tiene propuestas de cooperación local-global que buscan derroteros alter-

\footnotetext{
${ }^{3}$ Organización de Naciones Unidas, Resolución 55/2, Nueva York.
} 
nativos para la globalización, fundados en la solidaridad, la equidad, la reciprocidad y la justicia.

\section{CONCLUSIONES}

La FAO ha propiciado el desarrollo de mecanismos de gobernanza a nivel global en torno a la agricultura y la alimentación que han implicado la validación de ciertas formas de entender y actuar sobre esta materia, el establecimiento de acuerdos y formas de cooperación y coordinación de instituciones públicas y privadas nacionales e internacionales y el desarrollo y articulación de redes de relaciones - que abarcan a grupos y a organizaciones no gubernamentales con capacidad de actuar local y globalmente sobre la producción, distribución y consumo de alimentos- L La FAO, no obstante su lenguaje apolítico y técnico, adopta ciertas ideologías del desarrollo para definir, evaluar y justificar sus propuestas de política agroalimentaria mundial. Esta selección se realiza, por una parte, en un determinado contexto político global en el que entran en juego múltiples intereses públicos y privados y en los que los recursos económicos y las posibilidades de acción que permiten a los gobiernos se encuentran marcadamente diferenciados a nivel global; por ello, no son sólo los criterios científicos y racionales los que dan sustento a las propuestas de la FAO. Por otra parte, la adopción de una determinada ideología se realiza en una arena de lucha, en la que otros grupos y organizaciones disputan la hegemonía de la ideología dominante en la FAO, al tiempo que le critican dar poca importancia al combate a las causas históricas y estructurales del hambre y de la pobreza en el mundo y le demandan una postura más frontal frente a las empresas trasnacionales y las instituciones financieras y de comercio internacional, las cuales tienen gran injerencia y responsabilidad en la situación que guarda la SA global.

Finalmente, el debate ideológico y político sobre la problemática agroalimentaria mundial se fundamenta en principios éticos, por lo que la alimentación se plantea como un derecho de todos los seres humanos. Esta propuesta retoma elementos de los análisis de la FAO sobre la SA, pero va más allá al exigir el cumplimiento, la trans- parencia y la rendición de cuentas de los gobiernos en el aseguramiento de este derecho universal a una alimentación suficiente, nutritiva y sana. En esta perspectiva se han acordado y justificado metas más precisas a nivel internacional y nacional, que tienen como propósito disminuir sustantivamente el hambre y la pobreza; pero sobre todo, se han articulado y coordinado redes que integran a grupos y a organizaciones no gubernamentales que actúan local y globalmente en contra del hambre y a favor de un desarrollo agroalimentario sustentable que preserve los derechos de las generaciones futuras.

\section{Bibliografía}

Aranda J. y C. Morales, 2002, "Evaluación de los beneficios actuales y el potencial para el combate a la pobreza de la participación en redes de comercio justo de café en la Coordinadora Estatal de Productores de Café de Oaxaca", Fair Trade Research Group, en $<$ http://www.colostate. edu/Depts/Sociology/FairTradeResearchGroup/doc/cep coreportfinal.pdf>, consultado el 22 de agosto de 2007.

Barkin, D., 2001, "Superando el paradigma neoliberal: desarrollo popular sustentable", en N. Giarracca (ed.), ¿Una nueva ruralidad en América Latina?, CLACSO, Buenos Aires.

Bonnal, P., B. Losch y S. Bainville, 2000, "Points de repères sur la multifonctionnalité de l'agriculture: les dimensions nationales et internationales du débat", ponencia presentada en el seminario "La multifonctionnalité de l'agriculture et la mise en place des contrats territoriaux d' exploitation (CTE) dans les départements d'outre-mer", 21-24 de noviembre, Bouillante, Guadalupe.

— , P. M. Bosc, J. M. Díaz, B. Losch, 2003, “'Multifuncionalidad de la agricultura' y 'Nueva Ruralidad'. ¿ Reestructuración de las políticas públicas a la hora de la globalización?", ponencia presentada en el seminario internacional "El mundo rural: transformaciones y perspectivas a la luz de la nueva ruralidad", Universidad Javeriana, CLACSO, REDCAPA, Bogotá.

Di Iacovo, 2003, "New Trenes in the Relationship between Farmers and Local Communities in Tuscany", en G. Van Huylenbroeck y G. Durand (eds.), Multifuntional Agriculture. A New Paradigme for Europeal Agriculture and Rural Development, Ashage, Inglaterra.

Durand, G. y G. Van Huylenbroeck, 2003, "Multifuncionality and Rural Development: A General Framework", en G. Van Huylenbroeck y G. Durand (eds.), Multifun- 
tional Agriculture. A New Paradigme for Europeal Agriculture and Rural Development, Ashage, Inglaterra.

Echeverri, R., 2006, "Desarrollo territorial sustentable, el camino político hacia la construcción territorial”, Revista de la Asociación Latinoamericana de Sociología Rural, núm. 3, pp. 199-222.

- y M. Ribero, 2002, Nueva ruralidad: visión del territorio en América y el Caribe, IICA, San José de Costa Rica.

FAO, 1975, Report of the World Food Conference, Roma, 5-16 de noviembre de 1974, ONU, Nueva York.

, 1996, "The Rome Declaration on World Food Security”, Population and Development Review, vol. 22, núm. 4, pp. 807-809.

_ 2002, Informe de la Cumbre Mundial sobre la Alimentación: cinco años después. Roma, en <http://www.fao. org/DOCREP/MEETING/005/Y7106s/Y7106S>.

Falcon, W. y L. Naylor, 2005, "Rethinking Food Security for the Twenty-First Century”, American Journal of Agricultural Economics, núm. 5, pp. 1113-1127.

Fritscher, M., 2004, "El tema agrícola en las disputas multilaterales: fracasos en la OMC", en M. del Valle (ed.), El desarrollo agrícola y rural del tercer mundo en el contexto de la mundialización, UNAM, Plaza y Valdés, México.

González, A., 2002, "Evaluación de los beneficios actuales y el potencial para el combate a la pobreza de la participación en redes de comercio justo de café", Fair Trade Research Group, en <http://www.colostate.edu/Depts/ Sociology/FairTradeResearchGroup/doc/almaCorregid o2005.pdf>, consultado el 22 de agosto de 2007.

_ 2004, "El Barzón: Neoliberal Policies and New Social Movements in Mexico", en G. Otero (ed.), Mexico on Transition. Challenging Neoliberal Globalism, Zed Books, Londres.

__ 2005, "Estado, desarrollo y organizaciones locales de productores en México", en J. Barvosa y G. Neiman (eds.), Acerca de la globalización en la agricultura. Territorios, empresas y desarrollo local en América Latina, CICCUS, Argentina.

Gordillo, G., 2003, “Cambio y riesgo: la agricultura familiar en un mundo globalizado", ponencia presentada en el taller "La importancia del enfoque territorial en el desarrollo rural de América Latina y el Caribe”, FIDA, FAO, BID, Milán.

Grindle, M. S., 1986, “The State and Agrarian Change”, en The State and the Countryside: Development Policy and Agrarian Politics in Latin America, The John Hopkins University Press, Baltimore y Londres, pp. 11-24.

Losch, B., 2002, "La Multifonctionnalité face aux défis des agriculteurs des Suds: une perspective de refondation des politiques publiques?", ponencia presentada en el co- loquio “La multifonctionnalité de l'activité agricole et sa reconnaissance par les politiques publiques", 21-22 de marzo, SFER, París.

—_ 2004,"The Debate on the Multifunctionality of Agriculture: From Trade Negotiations to Development Policies by the South", Journal of Agrarian Change, vol. 4 , núm. 3, pp. 336-360.

Maxwell, S., 1996, "Food Security: A Postmodern Perspectiva”, Food Policy, vol. 21, núm. 2, pp. 155-170.

Mechlem, K., 2004, "Food Security and the Right to Food in the Discourse of the United Nations", European Law Journal, vol. 10, núm. 5, pp. 631-648.

Mendoza, A., 2000, "Prospectiva de la agricultura mexicana”, ponencia presentada en el diplomado sobre seguridad nacional, Centro de Investigaciones sobre Seguridad Nacional, México.

Nigh, R., 1997, “Organic Agriculture and Globalization: A Maya Associative Corporation in Chiapas, Mexico", $\mathrm{Hu}$ man Organization, vol. 56, núm. 4, pp. 427-436.

Phillips, L. y S. Ilcan, 2003, "A World Free from Hunger: Global Imagination and Governance in the Age of Scientific Management”, Sociologia Rurales, vol. 43, núm. 4, pp. 434-453.

Pottier, J., 1999, Anthropology of Food. The Social Dynamics of Food Security, Polity Press, Cambridge.

Roseberry, W., 1994, "Hegemony and the Language of Contention", en G. Joseph y D. Nugent (eds.), Everyday Forms of State Formation. Revolution and the Negotiation of Rule in Modern Mexico, Duke University Press, Londres.

Smith, M., J. Pinting y S. Maxwell, 1992, "Household Food Security, Concepts and Definitions: An Annotated Bibliography", Development Bibliography, vol. 8, University of Sussex, Brighton.

Sepúlveda, S., 2002, El concepto de desarrollo rural sostenible y la gestión del desarrollo desde una perspectiva territorial, NEAD, Brasilia.

Torres, F., 2003, "La visión teórica de la seguridad alimentaria como componente de la seguridad nacional”, en F. Torres (ed.), Seguridad alimentaria: seguridad nacional, UNAM, Plaza y Valdés, México.

— - y T. Aguilar, 2003, "Aspectos de la vulnerabilidad alimentaria de México”, en F. Torres (ed.), Seguridad alimentaria: seguridad nacional, UNAM, Plaza y Valdés, México.

VanderHoff, F., 2002, "UCIRI, Unión de Comunidades Indígenas de la Región del Istmo", Fair Trade Research Group, en <http://www.colostate.edu/Depts/Sociology/ FairTradeResearchGroup/doc/UCIRI_final.pdf $>$, consultado el 22 de agosto de 2007. 